\title{
Restricciones en el canal de comunicación y representación de influencia en situación de \\ toma de decisión
}

\author{
Jorge R. Vivas ${ }^{1}$ \\ Nancy Terroni
}

\begin{abstract}
Resumen
La comunicación mediada por computadora ha tenido un efecto de alto impacto social en la última década. Los efectos psicosociales de la utilización de estos medios tecnológicos en la comunicación humana son observados con creciente interés. Este trabajo reporta las variaciones de la percepción de influencia durante una tarea de toma de decisión colaborativa al modificar las restricciones del canal de comunicación (presencial o mediado por computadora). Se trabajó con cien sujetos repartidos en ambas condiciones de tarea sobre un problema de decisión múltiple. Los análisis muestran una diferencia notable en los aspectos considerados por los individuos para la construcción de la representación de influencia en una y otra modalidad. Estas diferencias son interpretadas como producto de que la magnitud de la presencia social esta modulada por las restricciones inherentes al canal mediador, dificultando y hasta impidiendo, la percepción de los mecanismos de influencia social que permiten la construcción de una representación de influencia ajustada a los efectivos intercambios realizados en torno al producto generado colaborativamente.

Palabras clave: Representación de Influencia; Comunicación mediada por computadora; Cara a cara; Toma de decisión.
\end{abstract}

\section{Restrictions in the communication channel and influence representation in taking up decisions}

\begin{abstract}
Computer mediated communication had a high social impact in the last decade. The psychosocial effects of the use of these technological media on human communication are observed with growing interest. This work reports the differences of influence perception during the collaborative making decision task when varying the communicational channel restrictions (Face To Face or computer mediated). The design included a hundred students in both task conditions on a multiple decision problem. The outcomes show a great differences in how individuals remarks different aspects for the representational construction of the influence in each modality. These results suggest that the amount of social presence is modulated by the channel restrictions, which make it difficult and even constrain, the perception of the social mechanisms of influence that allow the adjusted influence of a representational construction to the real interchanges around the group product goal.

Keywords: Influence Representation; Computer mediated communication; Face to face; Make decision.
\end{abstract}

\section{Introducción}

Los procesos de influencia entre el individuo y su entorno han sido, desde la década de los 60 , uno de los temas dominantes de la Psicología Social. Su estudio se ha centrado en diferentes aspectos que articulan la compleja urdimbre que constituye la dimensión social del individuo humano.

Las primeras investigaciones en América se desarrollaron en torno al modo en que el contexto moldea y configura el comportamiento del individuo. Se privilegió, entonces, el estudio de la influencia mayoritaria y el conformismo social (Asch, 1951, 1956; Sherif \& Sherif, 1969). En los años 70, desde Europa y de la mano de Serge Moscovici, se comenzó con el estudio sistemático de las influencias en sentido inverso. Resultó, entonces, privilegiado el estudio de la innovación y la influencia minoritaria (Doise \& Moscovici, 1969; Moscovici, 1976), con particular énfasis en aquellos casos

${ }^{1}$ Endereço para correspondência:

Funes 3280, cuerpo 5, nivel 3, 7600 - Mar del Plata, Argentina.

E-mail: jvivas@mdp.edu.ar en los que el grupo se desvía de la norma y, por lo tanto, la tendencia es hacia la innovación social, elaborando de este modo, una teoría sobre la influencia social de las minorías activas. Es también Moscovici (1984), quien instala en la agenda de discusión de los temas relevantes de la Psicología Social el concepto de que los seres humanos generan representaciones, no tanto sobre la base de procesos de razonamiento o computaciones mentales, sino como a través de la comunicación y el actuación sobre los otros o sobre el mundo.

La influencia social ha sido definida como el proceso en el que la conducta de una persona tiene el efecto de cambiar la forma en la que otra persona se comporta, siente o piensa sobre determinado estímulo (Zimbardo \& Leippe, 1991). Este cambio genuino en las preferencias y comportamientos de un individuo o grupo a instancias de acordarlas con otros (Vander Zanden, 1990) ha sido profusamente estudiado por medio de un nutrido conjunto de diseños de investigación que variaron sistemáticamente las condiciones experimentales. Así, se estudió la influencia social en función de la naturaleza de la tarea desarrollada, o bien modificando el tamaño del 
grupo o las características situacionales de la actividad, operando sobre la incertidumbre y la confianza y, más recientemente, aplicando distintos niveles de restricción sobre el canal de comunicación utilizado.(Kaplan, 1989; Cummings et al., 1974; Laughlin et al., 1975; Yetton \& Bottger, 1983; Arkes et al., 1987; Zarnoth \& Sienezek, 1997; Kiesler \& Sproull, 1991; Peiró et al., 1993; Orengo et al., 1996).

Buena parte de la investigación psicosocial en Comunicación Mediada por Computadora se ha concentrado en las consecuencias del filtrado de las claves no verbales de la comunicación en el correo electrónico. Se observó que se reduce la presencia social como producto de las restricciones en el contacto visual, los gestos, las señales de aprobación o los silencios y dudas antes de contestar. En el correo electrónico no hay ninguna señal contextual, los participantes no pueden ver la distribución de las sillas para identificar uniones y rupturas, ni ubicar sitios de encuadre que permitan identificar la importancia o el patrocinio de las reuniones. Se ha descrito que esta ausencia relativa de señales desarrolla un lenguaje desinhibido, gestión negativa de conflicto, dificultades en la coordinación y la retroalimentación, problemas para alcanzar consenso y polarización del grupo (Kiesler et. al., 1984; Hiltz, et. al., 1986; Siegel, et al., 1986; Dubrovsky, et al., 1988; Harasim \& Winkelmans, 1990; Goode \& Johnson, 1991; Kiesler \& Sproull, 1991; Peiró et. al., 1993). Para ciertos autores que han estudiado los procesos de consenso e influencia social se habla de auténtica influencia cuando en un vínculo direccional un sujeto determina que la transmisión se produzca pero la misma no es controlada por el receptor (Wasserman \& Faust, 1994).

Una revisión de los modelos de influencia social realizada por Rice (1993) pone de manifiesto que tres componentes esenciales se encuentran generalmente presentes en todos los estudios para dar cuenta de los factores que facilitan o inhiben los procesos de influencia. Estos componentes son: a) La ambigüedad, incertidumbre o novedad de la situación que se presente; b) La conectividad, la proximidad social y fortaleza del vínculo con los otros, fuentes y objeto de influencia; y c) el prestigio o valor otorgado al mensaje del otro, tanto en lo relativo a su posición formal como en la atribución de relevancia eventual y temática.

Desde los primeros desarrollos Sherif \& Sherif (1969) mostraron que la ambigüedad de la situación estímulo propicia en el individuo una condición favorable a la influencia. De modo que la experiencia o conocimiento previo otorga al individuo una certidumbre que lo hace menos vulnerable a la influencia. La conectividad, por su parte, es una apreciación del monto de exposición a la influencia de los otros y lleva implícita la idea de que los individuos son sensibles a las opiniones, actitudes y comportamientos de quienes se hallan relacionados con ellos. El prestigio o valor otorgado a los otros, que se ha tematizado como la tercera fuente de influencia, se basa en el impacto de las actitudes, opiniones y conductas de los otros en nuestras opiniones, actitudes y decisiones. Factores como status, confianza, credibilidad, etc, son atributos que facilitan o inhiben estos procesos de influencia.

En este trabajo la ambigüedad del problema se neutralizó seleccionando un reactivo de baja opinabilidad (Supervivencia en la Luna de Hall \& Watson (1970) y que responde adecuadamente a los cuatro criterios de demostrabilidad de Laughlin \& Ellis (1986). De este modo se utilizó una tarea decisional del tipo de las denominadas intelectivas (Laughlin et al.,1975), esto es, problemas con estado final bien definidos (Simon, 1978) donde la argumentación de los participantes tiene un peso relevante en los procesos de interacción en pos del objetivo y la opinabilidad resulta reducida. La tarea fue calificada como intelectiva de acuerdo al grado de cumplimiento de los cuatro criterios de demostrabilidad: existe un sistema conceptual para caracterizar la tarea y hay consenso sobre las reglas propias de ese sistema; existe suficiente información disponible para resolver el problema, bien en el contexto de decisión o bien en la memoria; los miembros con respuestas tentativas erradas deben poseer una comprensión del sistema lo suficientemente acabado como para reconocer una respuesta correcta cuando se les es explicada; y al menos un miembro con una respuesta tentativa correcta debe disponer de suficiente tiempo, capacidad y motivación para explicar la respuesta correcta al resto del grupo.

Los individuos antes de participar de una situación interactiva, disponen de una serie de conocimientos, evaluaciones y marcos de referencia de sí mismos y de los otros (a veces genérico) con los que se ha de realizar la interacción. Un aspecto importante de esta representación es la que recorta la participación personal y la de los otros, en términos de la influencia percibida en la construcción del resultado grupal.

El presente trabajo reporta los resultados de explorar la modulación que producen las restricciones del canal de comunicación en la percepción y posterior construcción de la representación de participación e influencia. El análisis se realizó siguiendo la evolución de las modificaciones de opinión, la conectividad y flujo comunicacional y la centralidad y prestigio de los integrantes, en el marco de una tarea de toma de decisión colaborativa. 


\section{Método}

Se utilizó el ejercicio de toma de decisión colaborativa denominado Supervivencia en la Luna de Hall \& Watson (1970). El criterio utilizado para su selección consideró que por su naturaleza es un ejercicio cognitivamente atractivo, adecuado al nivel de competencia intelectual de los participantes y que como problema de toma de decisión dinámico se ubica como una tarea decisional de tipo intelectiva, de acuerdo a los cuatro criterios de demostrabilidad de Laughlin \& Ellis (1986), lo cual disminuye el peso de la asertividad del discurso y permite discriminar mejor la relevancia del conocimiento previo y la argumentación en los procesos de influencia social.

Participantes: 100 alumnos de tercer año de la carrera de Psicología de la Universidad de Mar del Plata, cursantes regulares de las asignaturas Teorías del Aprendizaje, Psicología Cognitiva y Psicología Educacional. Los grupos se compusieron de cinco miembros.

Diseño: Es similar para ambas modalidades de comunicación. La tarea consiste en un problema de ordenamiento de alternativas con decisión múltiple. Sobre el supuesto de una accidente imaginario durante el alunizaje en la nave que lo transporta, propone seleccionar entre quince elementos que no fueron deteriorados un orden de prioridades para llegar a la base madre, en función de su contribución a la supervivencia. Tras un primer momento de resolución individual se conforman al azar grupos de cinco miembros que en forma colectiva producen un nuevo ordenamiento, consensuando las propuestas individuales. En una tercera fase se solicita la ordenación definitiva individual. El problema tiene una solución óptima definida por un panel de expertos de la NASA (Hall \& Watson, 1970) que se entrega como clave de corrección a los participantes, como así también una guía de los cálculos elementales que se pueden realizar sobre la producción individual y la del pequeño grupo. Finalmente, se administra una escala a los participantes explorando la representación de influencia en la construcción del producto grupal.

El mismo diseño se utilizó en la modalidad mediada, sólo que para el agrupamiento e interacción electrónica en grupos de cinco, se instaló un canal privado de chat utilizando MIRC con cinco máquinas horizontalmente interconectadas y una sexta máquina de comando y registro de la actividad de la red ubicada en otra dependencia. Los participantes podían tanto interactuar en forma conjunta con los otros cuatro miembros del grupo (sale lo mismo en las cinco pantallas) como podían tener intercambios privados de pares no visibles para los otros tres integrantes del grupo.
Materiales: Los ítems utilizados para su ordenamiento pueden verse en el Anexo 1. Se confeccionaron hojas de registro de la dinámica grupal para la modalidad cara a cara, donde se consigna la frecuencia, dirección y sentido de la comunicación entre los miembros del grupo. En la modalidad mediada se dispone también del registro de los movimientos conservados en la un archivo de log.

Procedimiento: El procedimiento es semejante para las dos modalidades experimentales en que se desarrolló la tarea.

Consigna 1: (individual). "Usted pertenece a un grupo de astronautas, tiene la misión de encontrarse con la nave madre sobre la superficie iluminada de la luna. A causa de dificultades técnicas, su nave tuvo que alunizar a $300 \mathrm{Km}$ de la nave madre. Durante el descenso, gran parte del equipo de bordo quedó destruido. Sólo puede llevar consigo lo imprescindible para superar esa distancia. A continuación hay una lista de 15 cosas que no fueron dañadas. Su tarea consiste en hacer un ordenamiento de los objetos que deben ser llevados por la tripulación en la planilla adjunta. Otorgue el número 1 a la posición más importante, 2 a la que le sigue, etc. hasta que todas las posiciones estén ordenadas según ese criterio".

Consigna 2: En este tramo del ejercicio la propuesta es coordinar sus puntos de vista con los del resto de la tripulación. Ahora el grupo deberá ponerse de acuerdo para establecer un único ordenamiento de los elementos a trasladar. Para obtener consenso se deberán respetar las siguientes reglas:

Evite imponer a los otros su decisión personal; argumente con lógica.

Evite ceder sólo para obtener la unanimidad o para eludir conflictos; apoye otros puntos de vista sólo si usted está de acuerdo con ellos al menos en parte.

Evite técnicas de solución de conflictos como, por ejemplo elección de la mayoría, cálculo del término medio o transacción.

Considere las opiniones discrepantes como contribuciones útiles, en vez de sentirlas como perturbadoras.

Consigna 3: Ud. ha resuelto individualmente el ejercicio y luego intercambió puntos de vista con un grupo hasta llegar a una solución general consensuada. Probablemente algunas de sus elecciones individuales han sido modificadas por el grupo y, recíprocamente, su opinión habrá influenciado en otros colegas. Le pedimos ahora que, en forma individual, nos presente el 
ordenamiento que para Ud., definitivamente, es el que mejor se ajusta al problema planteado.

Consigna 4: En una escala de cinco intervalos entre mucho y nada cada participante deberá evaluar la siguiente cuestión "En tu opinión que influencia ha ejercido cada uno de los miembros del grupo sobre las decisiones que se han tomado" (Zornoza et al.,1993)

\section{Definición operativa de las variables}

1) Variables de influencia expresadas por cambio de opinión:

- Calificación inicial (D1): Sumatoria de la divergencia en términos absolutos entre la valoración individual inicial VI (1) y la Clave. De acuerdo a la propuesta de Bottger \& Yetton (1988) esta medida expresa el conocimiento previo sobre la tarea.

- Cambio de opinión individuo - grupo (V1PG): Sumatoria de la divergencia en términos absolutos entre la VI (1) y el Pequeño Grupo. Es una medida de la contribución individual al producto grupal (Zornoza et al., 1993) y representa aceptación grupal de lo propuesto.

- Cambio de opinión grupo - individual final (PGV2): Sumatoria de la divergencia en términos absolutos entre la VI (2) y el Pequeño Grupo. Es una medida del grado de conformidad que el individuo tuvo con el producto grupal.

- Cambio de opinión entre la valoración inicial y final (V1V2): Sumatoria de la divergencia en términos absolutos entre la VI (1) y la VI (2). Es una medida de la influencia del grupo sobre el individuo

Representación de influencia (REPR) se evaluó por medio de una escala Likert de 5 puntos, con referencia tanto a la actuación de sí mismo como a la de sus compañeros.

2) Variables de influencia reticular: (en base a Borgatti et al., 1999)

Centralidad: Grado de centralidad de Freeman (1979).

Descripción: El número de vértices adyacentes a un vértice dado en un grafo simétrico es el grado de centralidad de ese vértice. Si la relación es direccional y posee magnitud el grado de entrada y salida consiste en la suma de los valores de los lazos. El grado normalizado de centralidad es el grado dividido por el máximo grado posible. En este trabajo se consideró como centralidad el grado normalizado de salida y como prestigio el de entrada. Ambas medidas reflejan rasgos de liderazgo durante la tarea.

Conectividad: Influencia. (Hubbell, 1965)

Descripción: El objeto de este tratamiento fue obtener medidas de influencia social en función de la conectividad. La medida utilizada enumera los posibles caminos de una longitud dada para todos los pares de nodos. Los caminos más largos contribuyen menos en términos de influencia.

\section{Resultados}

1) El primer tratamiento permite observar, para ambas condiciones de interacción, el grado de asociación entre las medidas de influencia obtenidas por los cambios de opinión del sujeto en las distintas fases de actuación y la representación de influencia (REPR).

Tabla 1. Coeficientes de correlación de Pearson entre medidas de influencia por cambio en la opinión y la Representación de influencia en la modalidad presencial.

\begin{tabular}{l|l|l|l|l}
\hline Cara a cara & REPR & V1PG & PGV2 & V1V2 \\
\hline REPR & 1,000 &,$- 545^{* *}$ &,- 238 &,$- 514^{* *}$ \\
& &, 000 &, 120 &, 000 \\
\hline V1PG & 1,000 &, 293 &, $772^{* *}$ \\
& & &, 053 &, 000 \\
\hline PGV2 & & 1,000 &, 058 \\
& & & &, 707 \\
\hline V1V2 & & & 1,000 \\
\hline
\end{tabular}

La representación de influencia (REPR) se asocia significativamente con dos medidas de influencia en la modalidad cara a cara. En forma directa con la contribución al producto grupal (V1PG), de modo que para el resto del grupo, cuanto mayor es la contribución al producto grupal de un individuo, mayor es la aceptación de lo propuesto y más destacada la percepción de su influencia. En forma inversa con la magnitud del cambio total (V1V2), de modo que cuanto menor es el cambio de opinión entre la decisión individual inicial y final, mayor es la percepción de influencia. Conviene recordar que la medida V1PG es de magnitud inversa por lo que la correlación negativa puede ser leída en el sentido descrito. 
Tabla 2. Coeficientes de correlación de Pearson entre medidas de influencia por cambio en la opinión y la Representación de influencia en la modalidad mediada por computadora.

\begin{tabular}{l|l|l|l|l}
\hline $\begin{array}{l}\text { Comunicación } \\
\text { mediada }\end{array}$ & REPR & V1PG & PGV2 & V1V2 \\
\hline REPR & 1,000 &,- 253 &,$- 357^{*}$ &,- 192 \\
& &, 077 &, 026 &, 241 \\
\hline V1PG & & 1,000 &, $368^{*}$ &, $788^{* *}$ \\
& & &, 021 &, 000 \\
\hline PGV2 & & & 1,000 &, 015 \\
& & & &, 929 \\
\hline V1V2 & & & 1,000 \\
\hline
\end{tabular}

En la modalidad mediada no se presenta asociación entre la representación (REPR), la contribución (V1PG) y el cambio total de opinión individual (V1V2). Sólo se observa una asociación moderada con la magnitud de cambio de opinión posterior a la acción grupal (PGV2). Correlación ésta que carece de significado psicológico, ya que cada miembro del grupo desconoce la respuesta dada por los otros en la fase de valoración final individual. También se observa que a menor aceptación grupal de lo propuesto mayor es el cambio posterior al volver a la instancia individual. Esta relación no se verifica en cara a cara.
Para ambas modalidades la contribución al producto grupal (V1PG) se asocia fuertemente en forma inversa con la magnitud del cambio total de opinión entre las dos instancias individuales. A menor aceptación grupal de lo propuesto mayor resulta el cambio de opinión individual como puede observarse en ambas tablas.

2) Se analizaron entonces a continuación las relaciones entre la representación de influencia (REPR), el conocimiento previo (D1), la aceptación grupal (V1PG) y el nivel de actividad y visibilidad individual expresadas por la centralidad (CENTRA) y el prestigio (PRESTI).

Tabla 3. Coeficientes de correlación de Pearson entre Representación de influencia, medidas de influencia por cambio de opinión y propiedades reticulares, en la modalidad presencial.

\begin{tabular}{l|l|l|l|l|l}
\hline Cara a cara & REPR & V1PG & D1 & CENTRA & PRESTI \\
\hline REPR & 1,000 &,$- 545^{* *}$ &,- 095 &, $586^{* *}$ &, $533^{* *}$ \\
& &, 000 &, 510 &, 000 &, 000 \\
\hline V1PG & & 1,000 &, $510^{* *}$ &,- 209 &,- 225 \\
& & &, 000 &, 144 &, 116 \\
\hline D1 & & & 1,000 &, 020 &,- 043 \\
& & & & 1,092 &, 764 \\
\hline CENTRA & & & & &, $918^{* *}$ \\
& & & & & 1,000 \\
\hline PRESTI & & & & & \\
\hline
\end{tabular}

Además de las asociaciones ya descriptas, se puede observar que existe una fuerte asociación entre la representación de influencia y el nivel de actividad del individuo como emisor de mensajes (Centralidad) y también con la visibilidad lograda como receptor de mensajes (Prestigio) La Centralidad y Prestigio no se asocian, por otra parte, con el conocimiento previo ni con la contribución al producto, en esta condición. El conocimiento previo de la tarea (D1), a su vez, no se asocia con la representación de influencia. Esto parece sugerir que no se percibe a quien más conoce. 
Tabla 4. Coeficientes de correlación de Pearson entre Representación de influencia, medidas de influencia por cambio de opinión y propiedades reticulares, en la modalidad mediada.

\begin{tabular}{l|l|l|l|l|l}
\hline $\begin{array}{l}\text { Comunicación } \\
\text { mediada }\end{array}$ & REPR & V1PG & D1 & CENTRA & PRESTI \\
\hline REPR & 1,000 &,- 253 &,- 130 &, $337^{*}$ &, 183 \\
& &, 077 &, 367 &, 017 &, 203 \\
\hline V1PG & & 1,000 &, $647^{* *}$ &,$- 334^{*}$ &,$- 306^{*}$ \\
& & &, 000 &, 018 & -031 \\
\hline D1 & & 1,000 &,$- 292^{*}$ &,$- 305^{*}$ \\
& & & & 1,040 &, 032 \\
\hline CENTRA & & & & &, $899^{* *}$ \\
& & & & & 1,000 \\
\hline PRESTI & & & & & \\
\hline
\end{tabular}

En la modalidad mediada por computadora sólo se observa una asociación moderada entre la representación de influencia y el nivel de actividad (Centralidad). La representación parece corresponderse, exclusivamente, con quien emite más mensajes en el canal de chat. La centralidad y prestigio sí se asocian, en forma moderada, con el conocimiento previo (D1) y la contribución al producto (V1PG).

En ambas tablas se puede observar que la contribución al producto se asocia fuertemente con el conocimiento previo para este tipo de problemas, como fue reportado por Orengo et al. (1996).

3) Finalmente, se exploró la relación entre la percepción de influencia y otra medida reticular basada en la cohesión de la red comunicacional durante la tarea para cada individuo (INFLUEN). Se complementa con medidas de influencia por cambio de opinión (V1PG, V1V2) y medidas de participación, centralidad (CENTRA) y prestigio (PRESTI).

Tabla 5. Coeficientes de correlación de Pearson entre Representación de influencia, medidas de influencia y propiedades reticulares de influencia, en la modalidad presencial.

\begin{tabular}{l|l|l|l|l|l|l}
\hline Cara a cara & REPR & V1PG & V1V2 & INFLUEN & CENTRA & PRESTI \\
\hline REPR & 1,000 &,$- 545^{* *}$ & $\begin{array}{l}-, 514^{* *} \\
, 000\end{array}$ &, $654^{* *}$ &, $586^{* *}$ &,$- 533^{* *}$ \\
\hline V1PG & & 1,000 & $\begin{array}{l}, 772^{* *} \\
, 000\end{array}$ &,- 220 &,- 209 &,- 225 \\
\hline V1V2 & & & 1,000 &,- 139 & -170 &,- 201 \\
\hline INFLUEN & & & & 1,000 &, $709^{* *}$ &, $515^{* *}$ \\
\hline CENTRA & & & & & 1,000 &, $918^{* *}$ \\
\hline PRESTI & & & & & & 1,000 \\
\hline
\end{tabular}

La representación de influencia (REPR) se halla asociada con todas las medidas que reflejan influencia en la modalidad presencial. Las medidas de influencia por cambio de opinión (V1PG, V1V2) y las medidas reticulares (INFLUEN, CENTRA y PRESTI), se comportan de modo tal que no presentan asociación alguna entre sí, salvo, naturalmente, las existentes al interior de cada subgrupo. 
Tabla 6. Coeficientes de correlación de Pearson entre Representación de influencia, medidas de influencia y propiedades reticulares de influencia, en la modalidad mediada.

\begin{tabular}{l|l|l|l|l|l|l}
\hline $\begin{array}{l}\text { Comunicación } \\
\text { mediada }\end{array}$ & REPR & V1PG & V1V2 & INFLUEN & CENTRA & PRESTI \\
\hline REPR & 1,000 & $\begin{array}{l}-, 253 \\
, 077\end{array}$ & $\begin{array}{l}-, 192 \\
, 241\end{array}$ &, $585^{* *}$ &, $337^{*}$ &, 183 \\
\hline V1PG & & 1,000 & $\begin{array}{l}, 788^{* *} \\
, 000\end{array}$ &,- 213 &,$- 334^{*}$ &,$- 306^{*}$ \\
\hline V1V2 & & & 1,000 &,- 135 &,- 272 &,- 277 \\
\hline INFLUEN & & & & 1,000 &, $532^{* *}$ &, 200 \\
\hline CENTRA & & & & & 1,000 &, $899^{* *}$ \\
\hline PRESTI & & & & & & 1,000 \\
\hline
\end{tabular}

La representación de influencia (REPR) sólo se asocia con las medidas reticulares de centralidad (CENTRA) e influencia (INFLUEN) en la modalidad mediada por computadora. La Centralidad y Prestigio, en esta modalidad, presentan una asociación leve pero significativa con la aceptación de lo propuesto. Esto parece sugerir que resulta más aceptado por el grupo quien más mensajes intercambia con el resto durante la sesión.

\section{Análisis de los resultados y conclusiones}

En la modalidad cara a cara la percepción que los miembros se forjaron de la participación de cada uno en el producto grupal aparece asociada en forma directa a cuánto contribuyó cada miembro a la conformación de ese producto. Se construyó una representación de mayor influencia en la medida que lo propuesto por un integrante fue hecho propio por el grupo y esto se asocia, a su vez, con el conocimiento previo que esa persona tenía del problema planteado.

Complementariamente, existe una asociación inversa entre los individuos que se percibieron como más influyentes en la producción grupal y aquellos que fueron más permeables a la opinión del resto del grupo. Se construyó una representación de mayor participación en el producto grupal coincidente con la de aquellos miembros que sostuvieron más decididamente sus opiniones en las diversas instancias de actuación. Conviene recordar aquí que si bien los participantes tuvieron ocasión de conocer la opinión previa individual de sus compañeros, así como los intercambios producidos en la interacción grupal, no tuvieron oportunidad de saber cual resultó la opinión final individual de cada participante. Por lo que se puedi que, para la condición presencial analizada, la flexibilidad mostrada por los integrantes en ajustar sus puntos de vista con los de sus compañeros de grupo es percibida como menor firmeza y convicción en las opiniones propias y, por lo tanto, con menor capacidad de influencia en la constitución del producto grupal.

En síntesis, en la condición presencial se percibió como con mayor influencia en la constitución del producto grupal a aquellos miembros que mayor firmeza tuvieron en sus opiniones y menos las cambiaron en las distintas instancias de actuación.

En la condición mediada por computadora no se verifican estos resultados. La representación de influencia sólo se asocia en forma leve con el cambio de opinión posterior al momento grupal, correlación que carece de significado psicológico, ya que los integrantes conocen la opinión individual inicial de cada uno y el consenso grupal al que han llegado, pero desconocen la valoración individual final que produjeron los otros en el momento en que se aplicó el instrumento de exploración de las representaciones.

La diferencia intermodal en los resultados obtenidos, puede ser interpretada como producto de las restricciones en la presencia social paraverbal (voz, entonación, gestos, etc) producidas por el canal mediador de la comunicación en la utilización del chat. Este filtrado de señales parece dificultar y hasta impedir, la percepción de los mecanismos de influencia social que permiten la construcción de una representación de influencia ajustada a los efectivos intercambios realizados en torno al producto generado colaborativamente. La menor presencia social en la comunicación mediada interfiere con la posibilidad de realizar una lectura más ajustada de los fenómenos psicosociales por parte de los miembros.

Complementariamente, la no aceptación grupal de lo propuesto por los miembros llevó a un incremento en la 
modificación de las opiniones individuales para ambas modalidades acentuándose esta asociación en la modalidad mediada, donde se verifica que a menor aceptación de lo propuesto más cambia el individuo al volver a la condición individual final, y mostrando un compromiso menor con la producción grupal inmediatamente antes consensuada. Ese resultado sugiere que las decisiones producidas utilizando un canal que empobrece la presencia social de los actores disminuye el compromiso de los mismos con los acuerdos alcanzados.

El segundo tratamiento buscó establecer si en la condición mediada la percepción de influencia podría hallarse asociada a otros indicadores de influencia distintos de los materializados por las modificaciones de opinión. Se exploró la relación de la percepción de influencia con el conocimiento previo y con el nivel de participación en la red comunicacional, tanto en su condición de emisor como de receptor.

Los resultados obtenidos en la modalidad presencial mostraron que existe una fuerte asociación entre la representación de influencia y las otras medidas. La influencia expresada por las opiniones del individuo hechas propias por el grupo, la actividad del individuo como emisor de mensajes (centralidad) y la visibilidad del individuo como receptor de mensajes (prestigio) se hallan asociadas en forma directa a la representación de influencia. No se verifica asociación con el conocimiento previo que posee el individuo. En la modalidad mediada por computadora, por el contrario, sólo se observa una asociación moderada con la centralidad.

$\mathrm{La}$ interpretación de esos resultados sugiere que en la construcción de la representación de influencia los individuos consideran no solo la modificación y firmeza de las opiniones personales en la interacción grupal, sino también el volumen de participación de cada miembro, tanto como emisor de opiniones hacia el grupo como en función de receptor de opiniones y comentarios del resto del grupo.

Esa asociación, que es muy marcada en la modalidad cara a cara, se diluye en comunicación mediada. La representación de influencia se vincula sólo en forma no muy notable con la cantidad de emisiones de cada integrante. El único índice percibido por los individuos para construir la representación de influencia en comunicación mediada parece ser la cantidad de emisiones producidas y recibidas por el compañero durante el chat.

El análisis de esos resultados parece sugerir que la constitución de la representación de influencia se circunscribe en la modalidad mediada, básicamente, a la participación verbal escrita en el proceso que requiere la actividad, expresado por la centralidad, no contemplando con igual fortaleza otros aspectos directamente vinculados con la tarea, como la calidad de la participación ni la magnitud de los cambios de opinión operados durante la misma. También indica que la magnitud de la contribución de cada miembro al producto grupal se asocia en forma directa al mayor conocimiento previo de la tarea para ambas modalidades. Estos resultados sugieren que, para tareas de toma de decisión de tipo intelectiva, más contribuye el que tiene mayor racionalidad argumentativa para defender sus puntos de vista frente al grupo.

El nivel de actividad, expresados por la centralidad y el prestigio en la condición mediada se asocian con el conocimiento previo, pero no es así en la condición presencial. Esto puede interpretarse como que la mayor participación en la condición mediada se relaciona con el mayor conocimiento de la tarea y que en la modalidad cara a cara, la mayor centralidad puede asociarse a otras cuestiones distintas a la racionalidad argumentativa de los participantes. La interpretación de estos resultados parece señalar que en la comunicación mediada por computadora el individuo con mayor conocimiento previo tiene mayor participación y contribuye más al producto grupal. Posiblemente la mayor incidencia de la racionalidad argumentativa en la dinámica grupal se explique por las restricciones impuestas por el canal a la comunicación no verbal (conviene recordar que la comunicación mediante el empleo del chat es de tipo escrita).

Se exploró, entonces, la plausibilidad de la suposición de que la representación de influencia en comunicación mediada se construya, fundamentalmente, en base a la percepción de conductas asociadas a la mera participación en el flujo comunicacional, no considerando en toda su magnitud otros aspectos que, como la asertividad del discurso o la lógica de los intercambios de opinión, constituyen factores esenciales para la influencia individual en el logro de un producto grupal. Se incluyó con este propósito además de las medidas de actividad del individuo en la red, el análisis de una medida de influencia propuesta por Hubbell (1965) calculada a partir de las distancias geodésicas y extraída por análisis de redes sociales (Borgatti et al., 1999). Se pudo observar una correlación fuerte entre la representación de influencia y la medida de influencia reticular para ambas modalidades.

Los resultados indican que para ambas modalidades las medidas de influencia por cambio de opinión, las de influencia reticular y las de actividad por centralidad y prestigio, se comportan de un modo diferente. En la comunicación presencial todas las variables parecen participar en algún grado de la percepción que tiene cada integrante de la influencia ejercida por cada uno en el logro del producto grupal. En la modalidad mediada 
se confirma que dicha representación se nutre, fundamentalmente, de la percepción de aspectos que hacen a la dinámica del flujo reticular, la participación y el entramado de cada integrante, en forma independiente de la intervención de los individuos en los procesos de negociación de significado puesta de manifiesto por medio de los cambios de opinión en cada instancia de actuación de la tarea.

La interpretación global de los resultados logrados en este trabajo parecen sugerir que la conformación de una representación se nutre de varios componentes en la modalidad presencial y sólo del nivel de actividad de los participantes y de la cohesión en comunicación mediada. En la modalidad presencial se construye la representación de influencia en torno a los contenidos intercambiados, la magnitud de los cambios de opinión, la participación, la referencia y la cohesión vincular establecida en la tarea. En la representación construida durante el uso de la comunicación mediada (chat), en cambio, la percepción parece restringirse casi con exclusividad al nivel de actividad de los miembros del grupo durante la interacción, generando, en consecuencia una representación de influencia empobrecida, centrada en la dinámica comunicacional durante la tarea y alejada de la efectiva contribución a la constitución del producto grupal .

\section{Referências Bibliográficas}

Arkes, H. R.; Christensen, C.; Lai, C. y Blumer, C. (1987). Two methods of reducing overconfidence. Organizational Behavior and Human Decision Processes, 39, 133-144.

Asch, S. (1951). Effects of group pressure upon the modification and distortion of judgement. En: Getzkow, H. Groups, leadership and men. Pittsburgh, Carnegie Press.

Asch, S. (1956). Studies on independence and conformity a minority of one against an unanimous majority. Psychological Monographs, 416.

Borgatti, S. P.; Everett, M. G. \& Freeman, L. C. (1999). UCINET 5 for Windows. Software for Social Network Analysis. Natick: Analytic Technologies. V 5.2.0.2.

Bottger, P. C.; Yetton, P. W. (1988). An integration of process and decision scheme explanations of group problem solving performance. Organizational behavior and buman decision processes; 1988 Oct. Vol 42 (2) 234-249)

Cummings, L. L.; Huber, G. P. \& Arendt, E. (1974). Effects of size and spatial arrangements on group decision making. Academy of Management Journal, 17, 46-75.

Doise, W. \& Moscovici, S. (1969). Approche et évitement de deviant dans des groupes de cohesion differente. Bulletin de Psychologie, 23. 522-525.

Dubrovsky, V.; Kiesler, S.; Sethna, B. (1988). Expected and unexpected effects of computer media on group decision making. Unpublished Paper, Committee on Social Science Research in Computing. Pittsburgh, PA: Carnegie Mellon
University.

Freeman, L. C. (1979). Centrality in social networks: conceptual clarification. Social Networks 1, 215 -239.

Goode, J., \& Johnson, M. (1991). Putting out the flames: The etiquette and law of e-mail. Online, Nov. pp. 61-65.

Hall, J.; Watson, P. (1970). The effects of a normative intervention on group Decision-Making performance. Human Relations, 23, 4, 299-317.

Harasim, L. M., \& Winkelmans, T. (1990). CMC scholarly collaboration. Knowledge: creation, diffusion, utilization, 11(4), 382-409.

Hiltz, R. S.; Johnson, K.; \& Turoff, M. (1986). Experiments in group decision making: Communication process and outcome in face-to-face versus computerized conferences. Human Communication Research, 13(2), 225-252.

Hubbell, C. H. (1965). An input - output approach to clique identification. Sociometry, 28, 377-399.

Kaplan, M. F. (1989). Task situational and personal determinants of influence process in group decision making. En E. J. Lawler \& B. Markovsky (Eds.) Advances in group process (Vol. 6) Greenwich, CT: JAI Press.

Kiesler, S.; Siegal, J.; \& McGuire, T. W. (1984). Social psychological aspects of computer-mediated communication. American Psychologist, 39 (10), 1123-1134.

Kiesler, S.; \& Sproull, L. (1991). Group decision making and communication technology. Organization, Behaviour and Human Decision Processes.

Laughlin, P. R. \& Ellis, A. (1986). Demonstrability and social combination processes on mathematical intellective tasks. Journal of Experimental Social Psychology, 22, 177-189.

Laughlin, P. R.; Kerr, N. L.; Davis, J. H.; Haljaff, H. M. \& Marciniak, K. A. (1975). Group size, member ability, and social decision schemes on an intellective task. Journal of Personality and Social Psychology, 31, 522-535.

Moscovici, S. (1976). Social influence and social change. London: Academic Press.

Moscovici, S. (1984). The myth of the lonely paradigm: a rejoinder. Social Research, 5, 4, 939-967.

Orengo, V.; Zornoza, A.; Acín, C.; Prieto, F. \& Peiró, J. M. (1996). Análisis de la Interacción Grupal a través de medidas de observación en comunicación mediada. Revista de Psicología Social, 11 (2) 129-256.

Peiró, J. M.; Prieto, M. \& Zornoza, A. M. (1993). Nuevas tecnologías telemáticas y trabajo grupal. Una perspectiva psicosocial. Psicothema, Vol 5, $287-305$.

Rice, R. (1993). Using Network Concepts to clarify sources and mechanisms of social influence. En: W. Richards, S.: Barnett, G. (eds.) Progress in communication sciences, vol.12

Sherif, M. \& Sherif, C. (1969). Social Psychology. New York, Harper and Row.

Siegel, J.; Dubrovsky, V.; Kiesler, S.; Mc Guire, T. (1986). Group process in computer mediated communication. Organizational Behavior and Human Decision Processes, 37 (2) 157-187.

Simon, H. A. (1978). Information Processing Theory of human problem solving. En W. Estes (Ed.) Handbook of learning and cognitive processes. Vol. 5: Human Information Processing. Hilsdale, NJ: 
Vander Zanden, J. W. (1990). Manual de Psicología Social. Buenos Aires: Paidos.

Wasserman, S. \& Faust, K. (1994). Social Network Analysis. Methods and Applications. Cambridge: Cambridge University Press.

Yetton, P. \& Bottger, P. (1983). The relationships among group size, member ability, social desicion schemes, and performance. Organizational Behaviour and Human Performance, 32, 145-159.

Zarnoth, P. \& Sienezek, J. A. (1997). The Social Influence of Confidence in Group Decision Making. Journal of Experimental Social Psychology 33, 345-366.
Zimbardo, P.; Leippe, M. (1991). The psychology of attitude change and social influence. N.Y. McGraw Hill.

Zornoza, A.; Orengo, V.; Salanova, M.; Peiro, J. M. y Prieto, F. (1993). Procesos de status, liderazgo e influencia en la comunicación mediada. En L. Munduate Jaca y M. Barón Duque (comp.) Psicología del trabajo y de las organizaciones. (pp. 101-114). Sevilla: Eudema.

Recebido em 16/04/2001

Revisado em 06/06/2001

Aceito em 21/06/2001

Sobre os autores:

Jorge R. Vivas é Licenciado em Psicologia. Professor de Psicología Cognitiva e Teorias da Aprendizagem. Diretor do Grupo de Pesquisa em Psicología Cognitiva e Educacional Faculdade de Psicología Universidade Nacional de Mar del Plata

Nancy Terroni é Licenciada en Psicología. Docente-Investigadora de la Facultad de Psicología, Universidad Nacional de Mar del Plata (UNMdP). Argentina -Becaria de Perfeccionamiento (2001-2002). Cátedra: Teorías del Aprendizaje.Grupo de Investigación: Psicología Cognitiva y Educacional. Cuenta con publicaciones y presentaciones en congresos nacionales e internacionales. 
Apellido y nombre

Anexo 1

\begin{tabular}{|c|c|c|c|c|c|c|c|c|c|c|c|c|}
\hline Objetos & $\mathrm{v} 1$ & pg & v1pg & v2 & pgv2 & $\mathrm{d} 1$ & v1v2 & Repr & Influ & Centr & Prest & Clave \\
\hline 1 Caja de fósforos & & & & & & & & & & & & \\
\hline $\begin{array}{l}1 \text { lata de comestibles } \\
\text { concentrados }\end{array}$ & & & & & & & & & & & & \\
\hline $\begin{array}{l}20 \text { metros de cuerda } \\
\text { de nylon }\end{array}$ & & & & & & & & & & & & \\
\hline $\begin{array}{l}30 \mathrm{~m}^{2} \text { de seda de } \\
\text { paracaídas }\end{array}$ & & & & & & & & & & & & \\
\hline 1 calentador portátil & & & & & & & & & & & & \\
\hline 2 pistolas $7,65 \mathrm{~mm}$. & & & & & & & & & & & & \\
\hline $\begin{array}{l}1 \text { lata de leche en } \\
\text { polvo }\end{array}$ & & & & & & & & & & & & \\
\hline $\begin{array}{l}2 \text { tanques de } \mathrm{O} 250 \\
1 .\end{array}$ & & & & & & & & & & & & \\
\hline $\begin{array}{l}1 \text { planisferio celeste } \\
\text { (constelación lunar) }\end{array}$ & & & & & & & & & & & & \\
\hline $\begin{array}{l}1 \text { bote neumático } \\
\text { con botellas de } \mathrm{co} 2\end{array}$ & & & & & & & & & & & & \\
\hline 1 brújula & & & & & & & & & & & & \\
\hline 20 litros de agua & & & & & & & & & & & & \\
\hline $\begin{array}{l}\text { cartuchos de señales } \\
\text { (arden también en } \\
\text { espacios sin aire) }\end{array}$ & & & & & & & & & & & & \\
\hline $\begin{array}{l}1 \text { botiquín de } 1 \text { ros } \\
\text { auxilios con agujas } \\
\text { para inyecciones. }\end{array}$ & & & & & & & & & & & & \\
\hline $\begin{array}{l}1 \text { transm - receptor } \\
\mathrm{fm} \text { con batería solar. }\end{array}$ & & & & & & & & & & & & \\
\hline totales & & & & & & & & & & & & \\
\hline
\end{tabular}

